(Aus der Universitäts Augenklinik zu Heidelberg.)

\title{
Über Heilungen von Netzhautablösung und die rheumatische Netzhautablösung.
}

\author{
Von \\ Professor L. Schreiber, \\ Heidelberg.
}

Mit der Diagnose "Netzhautablösung" ist nach Ansicht einer nicht geringen Zahl von Fachgenossen das Urteil baldiger oder späterer "Erblindung" des Auges gesprochen. Einzelne gehen in ihrem Pessimismus so weit, daß sie dem Kranken mit Netzhautablösung gleich bei der ersten Untersuchung das Auge als unrettbar verloren bezeichnen und daß sie ihm dringend raten, auch nicht einen Tag mit Behandlungsversuchen, die doch nutzlos wären, zu verlieren, sondern sofort ihre Arbeit in gewohnter Weise wieder aufzunehmen. - Recht charakteristisch in dieser Beziehung ist eine Arbeit von $\mathrm{Vail}^{1}$ ), die das Ergebnis einer Umfrage bei 460 der bekanntesten und beschäftigsten amerikanischen Augenärzte zusammenstellt. Von den 281 Antworten, die Vail erhielt, lauteten 250 , daB sie trotz mannigfacher operativer und nicht operativer Behandlung keinen einzigen Fall von Heilung einer (nicht traumatischen) Netzhautablösung gesehen hätten. Dabei berechnet Vail, daß die 250 Augenärzte eine Erfahrung an etwa 25000 Fällen von Netzhautablösung repräsentieren.

Aus statistischen Arbeiten deutscher Kliniker wissen wir, daß die Prognose der Netzhantablösung glücklicherweise nicht ganz so trostlos ist, wie sie nach obiger Statistik erscheint. So berechnet Th. Leber $\mathbf{r}^{2}$ - und zwar ohne Berücksichtigung der zum Teil erheblich besseren Erfolge der neuern operativen Behandlungsmethoden -, daß Heilungen mit gutem und brauchbaren Selvermögen in der Regel in $3-5 \%$ der Falle erzielt werden.

Aber auch dieses Ergebnis ist noch traurig genug und es zeigt, daß die Heilung einer Netzhautablösung als ein immerhin seltenes Ereignis zu betrachten ist. - Deshalb dürfte es trotz der schon vorhandenen einschlägigen Veröffentlichungen noch erwünscht sein, weitere Fälle von ge-

1) Vail, An inquiry into results of the established treatment of detachment of the retina, and a new theory. Annals of Ophthalmology, January 1913.

2) Graefe-Saemisch-Hess, Handb. d. ges. Augenheill. Bd. VII. T. II. B. 1607 . 
heilter Netzhautablösung bekannt zu geben. Das eingehende Studium einer möglichst großen Zahl gerade solcher Fälle ist vielleicht nicht der schlechteste Weg, um uns in der Exkenntnis der Ursache, der Entstehung und Heilungsmöglichkeit der Netzhautablösung weiterzuführen.

Den größten Teil des Yaterials, über das ich berichten möchte, entnehme ich der Dissertation von Seible ${ }^{1}$ ), der auf meine Veranlassung sämtliche Fälle von Netzhautablösung, die in den Jahren 1901-1912 in der Heidelberger Universitäts-Augenklinik stationär behandelt wurden, statistisch bearbeitet hat. In diesem Zeitraum kamen 163 Patienten zur Beobachtung, von denen $23(=14,1 \%)$ doppelseitig erkrankt waren; somit umfaßt die Statistik $163+23=186$ Augen mit Netzhautablösung. Heilung konnte bei 12 von $163(=7,4 \%)$ Patienten bzw. an 14 von $186(=7,5 \%)$ Angen beobachtet werden.

Sämtliche 163 Kranke wurden im Sommer 1914 zur Nachuntersuchung einbestellt und von diesen sind 84 Patienten unserer Aufforderung nachgekommen, die alle ron Seible und mir ge meinsa m genau bei weiter Pupille untersucht wurden: - Wenn meine Zahlenangaben von denen der Seibleschen Arbeit bie und da abweichen, so hat das darin seinen Grund, daB ich nach dem Jahre 1914 die Beobachtungen noch allein fortgesetzt und die geheilten Fälle von Netzhautablösung im Sommer 1918 einer nochmaligen Kontrolluntersuchung unterworfen habe. - $\mathrm{Da}$ die Seiblesche Dissertation vielen Lesern nur mit großer Mühe zugänglich sein dürite, zudem gelegentlich meiner späteren Untersuchungen nicht nur der objektive Befund, sondern in mehreren Fällen auch die Anamnese in wesentlichen Punkten ergänzt wurde, möchte ich im folgenden die Krankengesohichten trotz der bereits durch Seible erfolgten Veröffentlichung nochmals wiedergeben:

Fall T. 50jähriger Mann P. F. Aufnahme 12. V. bis 8. VT. 1903. Von Kindheit an stark kurzsichtig und stets mit beiden Augen schlecht gesehen. Derselbe bemerkte seit 10 Tagen Flimmern und dunkle Punkte wor dem rechten Auge. Anamnese im übrigen ohne Besonderheit, keine nennenswerte roraufgehende oder bestehende Allgemeinerlkankung.

Bei der Anfnahme 12. V. 1903.

$$
\begin{aligned}
& \mathrm{R}-15,0 \text { D. } S=5 / 25^{\circ} \\
& \mathrm{L}-8,0 \text { D. } \mathrm{S}=5 / 15^{\circ}
\end{aligned}
$$

Das Gesichtsfeld des rechten Auges war im temporalen unteren Quadranten auf $40^{\circ}$ eingeschränkt. Vorderer Bulbusabsehnitt ohne Besonderheit.

Ophthalmoskop: Rechtes Auge. In der Peripherie oben nasal großer h u feis enför miger Netzha u triß mit Ablösung der Netzhaut, die auf die nächste Lmgebung des Risses beschränkt ist. Die Ränder des Risses sind grauweiß getrübt; am unteren Rande eine kleine Blutung, in weleher ein kleines Gefä $B$ endet. In der

1) Seible, R., Klinisch-statistische Untersuchungen über Netzhautablösung auf Grund des Heidelberger Krankenmaterials 1901-1912. Inaug.-Diss. Heidel. berg 1915 . 
Macula lutea feine gelbliche alte chorioiditische Herde. Einzelne grobe und feinere flottierende Glaskörpertrübungen.

Behandlung. 6 wöchige klimische Behandlung mit subconjunkt, NaClInjektionen; Quecksilberimunctionskur, Jodkalium. - Bei der Entlassung 1903 war der ophtbalrnoskopische Befund im ganzen unverändert. - Sommer 1914 lag die Netzhaut überall glatt an.

$$
\begin{aligned}
& \text { Juli 1918. R. }-19,0 \text { D. } S=5 / 15 \text { o. Gl. } \frac{0,06}{0,40} \text {. } \\
& \text { L. }-11,0 \text { D. } S=5 / 15 \text { o. GI. } \frac{0,10}{0,40} \text {. }
\end{aligned}
$$

Das Gesichtsfeld des rechten Auges ist für ,Weiß ${ }^{\text {c }}$ bei gutem Tageslicht annähernd normal, bei herabgesetzter Beleuchtung temporal unten auf $65-70^{\circ}$ eingeengt; für "Blau" starke Einengung, temporal bis $23^{\circ}$, unten 20 bis $30^{\circ}$, oben $32^{\circ}$.

Ophtial moskop: Rechtes Auge: einzelne periphere Linsentrübungen; 2 grobe Glaskörpertrübungen. Großes temporales Staphyloma posticum. Feine gelbliche alte chorioiditische Herde in der Macula lutea. Peripher nasal oben an der Stelle der früheren Ablatio ret. sieht man den geheilten hufeisenförmigen Netzhautriß, dessen Ränder weit klaffen, so daß hier noch die Aderhaut in Hufeisenform gegenüber der Umgebung lebhaft rot hervortritt. Das zungenförmige Läppchen des Risses erscheint stark graulich getrübt und fast undurchsichtig. Unterhalb des Risses sieht man die Grenzlinie der ursprünglichen Ablösung in Form eines längeren Pigmentstreifens. Die Netzhaut liegt überall glatt an. Sonstige Veränderungen sind nicht vorhanden, nur bemerkt man temporal oben an der Grenze des Sichtbaren ausgedehnte alte chorioiditische Veränderungen.

Linkes Auge. Periphere Linsentrübungen. Staphyloma postic. und feine alte chorioiditische Veränderungen in der Macula wie rechts.

Zusammenfassung: Heilung einer umschriebenen Netzhaut. ablösung mit großem Netzhautriß im hochgradig myopischen Auge bei nichtoperativer Behandlung mit Sehschärfe $=1 / 3$.

Beobachtung l5. Jahre.

Fall II. 32 jähriges Fräulein K. Th. Aufnahme 23. IV. bis 16. V. 1903. Von jeher stark kurzsichtig. 3 Monate vor Eintritt in die Klinik plötzliche Sehstörung am rechten Auge. Sofort von Dr. Sulzer in Neustadt a. H. mit Bettruhe und Jodkalium behandelt. Anfangs Besserung, dann Rückfall; seit 2 Monaten ist das rechte Auge unverändert schlecht. - Seit frühester Kindheit schwächlich, litt angeblich an englischer Krankheit; hochgradige Blutarmut. Wiederholte Anfälle von Blinddarmentzündung (1904 operiert). 1907 und 1908 Kniegelenksentzündung, die sich 1917 wiederholte; davon abgesehen sind keine rheumatischen Erscheinungen nachweisbar.

Bei der Aufnahme 23. IV. 1903:

$$
\begin{aligned}
& \text { R. }-12,0 \text { D. } \mathrm{S}=\text { Finger zählen in } 3^{1 / 2} \text { M. o. Gl. } \frac{0,06}{0,60} \text {. } \\
& \text { L. }-14,0 \text { D. } \mathrm{S}=5 / 20 \text { o. Gl. } \frac{0,06}{0,30} \text {. }
\end{aligned}
$$

Gesichtsfeld des rechten Auges oben und nasal stark eingeschränkt, zentral besteht ein relatives Skotom; nur außen unten ist das Gesichtsfeld normal. Rechtes Auge: Der vordere Bulbusabschnitt ist normal; nur ist die rechte Pupille etwas weiter als die linke, reagiert aber gut.

Ophthalmosko pisch. In der oberen Hälfte des Fundus besteht eine ausgedebnte seichte Netzhautablösung, die etwa bis zur Mitte zwischen Papille und 
Aquator reicht, die Maoula lutea einschließend. Im untersten Drittel des Augengrundes ist die Ablösung blasig und wogend.

Behandlung. II subconjunctivale NaCl-Injektionen.

16. V. 1903. Nach 3 Wochen - Entlassung.

Ophthalmoskopisch. Rechtes Auge: Die Netzhant liegt überall glatt an.

$$
\text { R. }-12,0 \text { D. } S=\$ / 20 \text { p.; p. o. G1. } \frac{0,07}{0,40} \text {. }
$$

Eine Yachuntersuchung der Kranken in der Klinik lieb sioh leider nicht er. möglichen; aber am 27. IV. 1916 wurde dieselbe von dem erstbehandelnden Angenauzte Hern Dr. Sulzer eingehend untersucht, der mir hierüber freundlicherweise folgendes berichtete:

$$
\begin{aligned}
& \text { R. }-14,0 \text { D. } S=1 / 24 \frac{0,10}{0,30} . \\
& \text { L. }-16,0 \text { D. } S=0 / 24 \frac{0,10}{0,3} .
\end{aligned}
$$

Gesichtsfeld trei.

Ophthalmoskop isch. „Kejne Ablatio! „Das Auge sei all die Jahre gut geblieben." Es scheint also eine definitive Heilung qu sein."

Am 28. IX. 1918 bestätigte mir die nach Nen-Tuchel (Westpreußen) verzogene Patientin brieflich, daB sie mit dem rechten Auge - wie nach der Entlassung aus der Klinik - weitex gut sehe, trotzdem sie in den letzten 4 Jahren viel Näharbeit habe verrichten müssen.

Zu sammenfass ang: Heilung einer sehr angedehnten, stellenweise blasigen Netzhatablösung (ohne nachweisbaren Netzhautriß) in hoohgradig myopischen Ange bei nichtoperativer BehandIung mit Seh sehäre $=1 / 4^{*}$

Beobachtuig (augenärtich) $13 \mathrm{Jahre}$.

Fall III. 49jährige Frau E. An. Aufnahme 7. III. bis 13. IV. 1912. Etwa 1905 bemerkte Patientin, daß sie mit dem reohten Auge schlecht sehe; die Gegenstände erscheinen alle verkrümmt und verbogen. Seit 3 Tagen die gleiche Er. scheinung am li n ke n Auge unter starker Abnahme des Sehrermögens, indem sich vor das Auge eine Wolke legt. Schon seit 6 bis 7 Wochen ständig Funkensehen.

Bei der Aufnahme 7. III. 1912.

$$
\text { R. }-17 \mathrm{D} .=\text { cyl. }-2,5 \mathrm{D}, \mathrm{A} .0^{\circ} \mathrm{S}=\text { Fg. in } 4 \mathrm{nl} ; \mathrm{o} \text {. G], } \frac{0,08}{0,90} \text {. }
$$

L. Finger zäblen in $1 / 2 \mathrm{~m}$ exzentr.

Beiderseits Cornea obne Besonderheit; tiefe Vorderkanmer.

Ophthalmos lo pisch: Rechtes A uge. Staphyloma posticum temporale. In der Macula Iutea ausgedehnte Aderhautatrophie mit geringer Pigmentierung; Sklerose der Aderhautgefäße.

Linkes Auge: In der ganzen oberen Hälfte ist die Netzhaut abgelöst und zwar ist die Ablösung temporal blasig, im übrigen flach; dieselbe geht bis an die Papille heran. Breites Staphyloma posticum temporale.

Behandlung: 8 NaCl- (5\%) Injektionen. Bei der Entlassung am 13. IV. 1912 war die Netzhaut im linken Auge völlig angelegt. Nach 2 Jahren, Sommer 1914, war der Befund noch derselbe, das Gesichtsfeld auch für Blau normal und der Visus L. -15 D. O cyl. $-2,5$ D. A. $0^{\circ} \mathrm{S}=5 / 15$.

Letzte Untersuchung am ö. VII. 1918.

Ru -17 D. 0 cyl. $-2,5$ D. A. $0^{\circ} \mathrm{S}=$ Fingerzählen in $1 \mathrm{~m}$.

L. o. Gl. $\frac{0,08}{0,40} ;-15$ D. $=0 y 1 .-2,5$ D. A. $0^{\circ} S=5 / 15$. 
Gesichtsfeld des linken Auges für Weiß annähernd normal und zwar auch bei stark herabgesetzter Beleuchtung; dagegen für Blau allseitig eingeengt, oben und unten bis $20^{\circ}$.

Ophthalmoskop isch: Rechtes Auge. Glaskörper frei. Die Papille erscheint atrophisch graurot. Riesengroßes Staphyloma posticum, das ohne Grenze in die atrophische Macula lutea übergeht. SkJerose der Chorioidealgefäße.

Linke s A uge. Glaskörper frei. Großes ringförmiges Staphyloma posticum. Die Retina liegt überall glatt an und zeigt insbesondere auch in der oberen Hälfte keine Veränderung; die Chorioidealzeichnung ist oben ebenso gut sichtbar wie unten. In der Macula lutea dagegen sieht man netzartig zusammenhängende streifige gelbliche und kleine weißliche Chorioidealherde mit Pigmentierung.

Zusammenfassung: Heilung einer sehr a usgedehnten, stellenweise blagigen Netzhautablösung (ohne nachweisbaren NetzhautriB) im hochgradig myopischen Auge bei nichtoperatjver Behandlung mit Sebschärfe $=1 / 3$.

Beobachtung 6 Jahre.

Fall IV. 30jährige Frau A. R. Aufnahme 31. VIII. bis 10. X. 1905. Von jeher kurzsichtiy. Seit etwa 1 Jahre Elockensehen, seit etwa 5 Monaten starke Sehverschlechterung am rechten Auge. Seit etwa 4 Monaten Flockensehen am linken Auge ohne Abnahme des Sehvermögens. Anamnese im übrigen belanglos; Patientin ist sonst gesund.

Bei der Aufnahme am 31. VIII. 1905. Beiderseits vorderer Bulbusabschnitt ohne Veränderungen.' Rechtes Auge: Ziemlich zahlreiche feine fädige und membranöse flottierende Glaskörpertrübungen. Mäßig großes Staphyloma posticum. - In der unteren Hälfte ist die Retina stark faltig abgehoben; temporal ist auch fast der ganze obere Quadrant der Retina abgelöst. Temporal unten 2 kle ine Netzhatrisse.

Linkes A uge: Einzelne feine flottierende Glaskörpertrübungen; eine gröbere präpapilläre Trübung. Peripher unten seichte Netzhautablösung mit ausgesprochener Gefäßparallaxe. (Die Ablösung nimmt in den folgenden Wochen noch zu, die Netzhaut bleibt aber durchsichtig.)

$$
\text { R. }-3,0 \text { D. } S=5 / 50 \text {; o. Gi. } \frac{0,10}{0,40},
$$

Gesichtsfeld in der oberen Fälfte um $30^{\circ}$. eingeschränkt.

$$
\text { L. }-7,0 \text { D. } \mathrm{S}=5 / 7-5 / 5 \text {; o. Gl. } \frac{0,10}{0,30} \text {, }
$$

Gesichtsfeld in der oberen Hälfte um $20^{\circ}$ eingeschränkt.

Behandlung: Bettruhe, Sublimatpillen, NaCl-Injektionen (R. 7, L. 6).

Bei der Entlassung am 10. X. 1905 keine wesentliche Besserung.

1906 wurde eine Inunctionskur vorgenommen, ebenfalls ohne nennenswerten Erfolg. Bei einer viertägigen Beobachtung im Jahre 1910 fand sich am rechten Auge vorgeschrittene Katarakt, am linken Auge war die Netzhautablösung wicht mehr mit Sicherheit nachweisbar. - Bei der Nachuntersuchung 1914 unterlag es keinem Zweifel mehr, daß die Netzha ut im linken A uge ïberall glatt anliege.

Bei der letzten Untersuchung am 4. VII. 1918 war der Befund wie folgt:

Rechtes Auge: Cataracta complicata. Amaurose.

Linkes Auge: $-7,0$ D. $S={ }^{5} / 4 ;-5,0$ D. $\frac{0,3}{0,4}$; Gesichtsfeld für Weiß und Blau ziemlich gleichmäßig oben um $25^{\circ}$ eingeengt. Einzelne gröbere Glaskörpertrübungen. Unten an der Stelle der früheren Ablösung liegt die Netzhaut - wie auch sonst überall - glatt an; hier sieht man zahlreiche meist verzweigte schwarze 
Pigmentflecke und kleinere atrophische Aderhautherdc. Temporal unten ein großer mit scharfen Linien abgegrenzter entfärbter Bezirk, der verästelte Pigmentierungen aufweist.

Zusammenfassung: Doppelseitige Netzhatablösung bei mittelgradiger Myopie (ca, - 7,0 D.); nichtoperative Behandlung. Am rechter Auge sehr ausgedehnte Ablosung mit 2 Netzhantrissen; Ausgang in Cataracta complicata, Amaurose.

Am Jinken Auge Heilung einer amsehriebenen Netzhautablösung fohne nachweisbaren und wahrscheinlioh auch nicht vorhandenen NetzhautriB) mit Sehschärfe $5 / 4$.

Beobachtung 13 Jahre.

Fall V. 50 jähriger Mann T. H. Aufnahme 24. X. bis 15. XI. 1911. Patient, der schon 4 Jahre vorher (1907) wegen Keratitis parenchymatosa und Chorioiditis disseminata in Behandlung der Augenklinik stand, suchte die Klinik 1911 zum Zwecke der Invalidisierung auf und klagte bei dieser Gelegenheit über eine erhebliche Sehverschlechterung am linken Auge; am 9. VIT. 1910 war die Wass er mannsche Blutserumreaktion negativ.

Bei der Aufnahme am 24. X. 1911. Beiderseits reizlos. Die Cornea zeigt zarte diffuse graue alte Trübungen, die das o pthal mos ko pis che Bild verschleiern. Beiderseits großes Staphyloma posticum. Alte chorioiditische Herde.

Linkes Auge: Unmittelbar am nasalen Papillenrande beginnt eine Netzhautablösung, die sich nach unten ausbreitet und temporal unten am stärksten ist; hier bildet die abgehobene Netzhaut mehrere größere silbergraue Falten.

R. $-13,0$ D. $\mathrm{S}=4 / 50^{2}$

L. $\mathrm{S}=$ Hand bewegung in $1 / 2 \mathrm{~m}$ exzentrisch außen.

Behandlung: Links 7 subconjunctivale NaCl-Injektionen.

Bei der Entlassung - nach 3 Wochen - war die Netzhautablösung noch vorhanden, aber entschieden rückgängig; der gleiche Befund 4 Wochen später.

Sommer 1914 lag die Netzhaut, wie man sich trotz der Hornhauttrübungen einwandfrei überzengen konnte, ibberall glatt an.

R. $-15,0$ D. $\$=5 / 50 ; 2,25$ Sohrift,

I. $-15,0$ D. $S=5 / 50 ; 2,25$ Solurift.

Auf die sohriftliche Einbestellung zur Nachuntersuchung im Juli 1918 teilte die Ehefrau mit, daß Patient am 22. IX. 1915 gestorben sei und bis zuletzt mit dem linken Auge gut gesehen habe.

Zusammenfassung: Heilung einer auggedehnten Netzhautablösung (ohne nachweisbaren Netzhatriß) im hochgradig myopisehen Auge bei nichtoperativer Behandlungmit Sehschärfe $=1 / 10$. Bemerkenswert ist, daB Patient 4 Jahre vorher eine doppelseitige Keratitis parenchymatosa und Chorioiditis disseminata aberstanden hat.

Beobachtung 4 Jahre bis zum Tode (augenärztlich 3 Jahre).

Fall VI. 55 jährige Fran E. L. Aufnahme 6. III. bis 5. IV. 1902. Bisher angeblich gut geselen. Vor 12 Tagen Flimmern, Lichterscheinungen und Nebel for dem rechten Auge. Bei dieser Gelegenheit erst bemerkte Patientin, daß sie auch am linken Auge sehlecht sehe. - Von früheren Krankheiten sind bemerkenswert: im Alter von 9 Jahren eiternde Stelle am rechten Knie, die operiert werden mußte. Mit 50 Jahren rechtsseitige Lungen- und Brustfellentzündung, die ganz geheilt sein soll; kein Husten, aber häufiger Xachtschweiß. Von 6 Kindern sind 3 angeblich an Lungenkrankheit gestorben.

Bei der Aufnahme am 6. III, 1902. Rechte Lungenspitze suspekt auf Tuberkulose. Über dem rechten Knie mit dexn Knochen verwachsene Narbe. 
R. Fingerzählen in $2 \mathrm{~m}$; aus 3,0 Schrift einzelne Buchstaben erkannt.

L. Fingerzählen in $2 \mathrm{~m}$; aus 3,5 Schrift einzelne Buchstaben erkannt.

Rechtes Auge: Vorderer Bulbusabschnitt normal, abgesehen von einer feinen vorderen Polarkatarakt.

O phthalmoskop isch: Ziemlich dichte flottierende Glaskörpertrübungen. Totale Netzhautablösung; die Netzhaut, die nasal und oben grauweiß erseheint, überlagert fast die ganze Papille. In der unteren Hälfte ist die Ablösung flacher und die Netzhaut ist hier relativ durchsichtig. - Tonus gegen links etwas vermindert.

Linkes Auge: Vorderer Bulbusabschnitt normal. Ophthalmos ko pisch: Trübung am hinteren Linsenpol; mäBige Glaskörpertrübungen. Die Papille erscheint schmutzig graurötlich verfärbt. Die Netzhaut liegt überall an. Oberhalb der Papille bemerkt man ein langes, breites horizontal gelegenes Band von schwarzen Pigmentflecken; die Pigmentierung ist stellenweise zierlich, knochenkörperchenähnlich, nach außen aber klumpig (der Befund erinnert an eine wiederangelegte Netzhautablösung).

Behandlung: Zunächst Druckverband am rechten Auge; danach wird das Auge weich und es treten an der Cornea Faltentrübungen auf. Deshalb wird vom Verband abgesehen und Bettruhe, Hg-Inunktionskur angeordnet. AuBerdem 4 subconjunctivale NaCl-Injektionen.

Da die Netzhautablösung sich nicht besserte und sogar zunahm, wurde am 24. III. 1902 eine Skleral pu nk tio on oben nasal und am 2. IV. 1902 eine Skleralpunktion nasal unten ausgeführt.

5. IV. 1902 Entlassung. Rechts Netzhautablösung unverändert, total.

R. S. = Fingerzählen in $1 \mathrm{~m}$.

Eine Nachuntersuchung der Kranken war leider nicht möglich. Dagegen teilte uns der Sohn (der interessanterweise gleichfalls an einer Netzhautablösung erkrankt war, die zur Wiederanlegung kam - vgl. Fall VII) am 13. VII. $1918 \mathrm{im}$ Auftrage seiner Mutter mit, daß beide Augen nach der Entlassung aus der Klinik sich erheblich gebessert hätten, und zwar sei das rechte Auge das bessere. Patientin arbeitet nicht nur auf dem Felde und findet sich überall gut zurecht, sondern sie verrichtet Näharbeiten, bessert dem Sohn die Hosen aus und fädelt sich dabei selbst die Nadel ein.

Zusammenfassung: Dop pelseitige Netzhautablösung (ohne nachweisbaren Netzhautriß) unbekannter Ursache bei Emmetropie. Am linken Auge Spontanheilung. (Die Kranke wuBte von einer überstandenen Erkrankung dieses Auges nichts; der Befund der wiederangelegten Netzhautablösung wurde zufällig gelegentlich der Behandlung des anderen Auges erhoben.) Am rechten Ange wurde die Heilung der Netzhautablösung a ugenärztlich nicht beobachtet, dieselbe ist aber mit überwiegender Wahrschein lichkeit zu ersehlieBen: 1. aus den Angaben der Kranken, daB dieses Auge noch 16 Jahre nach der Ablösung sogar zu feinen Arbeiten gebrauchsfähig ist, 2, aus der.Tatsache dex Wiederanlegung einer Netzhautablösung am anderen Ange, 3. aus der interessanten Tatsache, daB auch der Sohn der Patientin an einer Netzhautablösung litt, die zur Ausheilung gekommen war (vgl. den folgenden Fall VII). - Da die Heilung zweifellos erst längere Zeit nach Abschluß der Behandlung aufgetreten ist, muB dieselbe als spontane betrachtet werden.

Beobachtung 16 Jahre.

Fall VII. 16 jähriger Knabe V. L. (Sohn von Fall VI). Aufnabme 15. IV. bis L. VI. 1905. Im Alter von 7 Jahren will Patient vor dem rechten Auge ,rote v. Graefes Arehiv für Ophthaimologie. Bd. 108. 
Flecke" gesehen haben; im Anschluß daran langsame Erblindung desseiben. Seit 5 Wochen am bisher gesunden und gut sehenden linken Auge allmählich zunehmende Sehversehlechterung mit Flimmern; hie und da links angeblich Schmerzen. Sonst gesund. Kein Anhaltspunkt für Rheumatismus, Lues usw.

Bei der Aufnahme am 15. IV, 1905:

Rechtes Auge: Blasse Cataracta complicata. Amaurose.

Linkes Auge: Vorderer Bulbusabschnitt normal. Glaskörper klar. In der oberen und unteren Netzhauthälfte mehrere flache und höhere Falten. Temporal unten schließt sich an eine solche Falte eine seichte Netzhautablösung von geringem Umfange an. Papille normal.

I. E. $S=5 / 35 \frac{0,25}{1,0}$ mühsam.

Gesichtsfeld temporal um $30^{\circ}$, oben um $25^{\circ}$ eingeengt.

Behandlung: Hg-Inunktionskur, Sublimatpillen; subconjunctivale NaClInjektionen.

Am 1. VI. 1905 Entlassung. Ophthalmoskopisch: Ablatio ret. unverändert.

L. $+0,75$ D. $S=5 / 15$ teilweise; $\frac{0,20}{0,60}$ mühsam.

15. V. 1916 ambulante Nachuntersuchung.

Rechtes A uge: unverändert.

Li nkes A uge: der Bulbus steht in Divergenz.

Ophthalmoskop isch: Feine spinngewebsartige und grobe Glaskörpertrübungen. Papille und Macula latea ohne Besonderheit. Mehrere weißliche bogige Netzhautstreifen in der nasalen Hälfte, ebensolche Streifen in der temporalen Hälfte oben and unten. Die Netzhautgefäße ziehen ohne wesentliche Knickung über die Streifen hinweg und zeigen auch sonst keine Veränderung. Zwei derartige Streifen temporal unten laufen zu einer V-Figur zusammen. Außerdem bemerkt man an verschiedenen Stellen lange Pigmentbogen. Der ganze Fundus zeigt feine retinale Pigmentierung und beim Blick ganz peripher unten sieht man große alte chorioiditisehe Herde.

L. o. G1. $S=2 / 24 ; 3,5$ Schrift.

Am 13. VIT. 1918. Ophthalmoskopischer Befund unverändert; die Netzhaut liegt überall glatt an. Skiaskop. L. $+3,0 \mathrm{D}$.

L. o. Gl. $S=2 / 30 ;+3,0$ D. $S=2 / 25+8,0$ D. 3,5 Sehrift.

Gesichtsfeld oben $\left(25-35^{\circ}\right)$, unten $\left(40^{\circ}\right)$ und besonders temporal $\left(20^{\circ}\right)$ stark eingeschränkt.

Zusammenfassung: Wahrscheinlich doppelseitige Netzhautab. lös ung (ohne nachweisbaren Netzhautriß) bei Hypermetropie. Ursache unbekannt, jedoch ist bemerkenswert ein familiäres Auftreten der Ablatio (vgl. Fall VI). Am rechten Auge Ausgang in Cataracta complicata. Am linken Auge Heilung der Netzhautablösung, die als S pontanheilung aufzufassen ist, da dieselbe zweifellos erst längere Zeit nach Abschluß der Behandlung erfolgte. Auf. fällige Analogie mit dem Verlaufe der Netzhautablösung bei der Mutter.

Beobachtung 13 Jahre.

Fall VIII. 22 jähriger Mann W. N. Aufnahme 11. X. bis 8. XI. 1912. Mit beiden Augen stets gut gesehen. - Am 10. X. 1912 flog dem Patienten beim Bohren ein Splitter ins linke Auge. 
Bei der Aufnahme am 11. X. 1912

Rechtes Ange: normal. E. $\mathrm{S}=5 / 4$.

Linkes Auge: Ciliarinjektion. Nasal oben punktförmige perforierende Cornealwunde; derselben gegenüber ein kleines Loch in der Iris; partielle Linsentrübung.

Ophthalmoskop isch: Im peripheren Teil des temporalen Abschnitts sieht man einen metallischen Splitter vor der Retina, dessen oberes Ende in die Retina eingespieBt ist. Temporal oben vor dem Splitter ist die Netzhaut abgelöst, als ob dieselbe von dem Splitter heruntergezogen wäre.

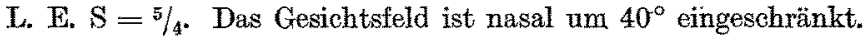

Behandlung: Sofortige Magnetextraktion. Fortschreitende traumatische Katarakt, die extrahiert wurde.

Nachuntersuchung 1914 (nach 2 Jahren).

Linkes Auge: Die Netzhaut liegt überall an; temporal oben sieht man an derselben hellrote strahlige Streifen und oberhalb dieser mehrere Pigmenthaufen. L. $+13,0$ D. S $=5 / 15$ teilweise.

Späteren Aufforderungen zur Nachuntersuchung leistete Patient keine Folge.

Zusammenfassung: Heilung einer umschriebenen traumatischen Netzhautablösung (ohne nachweisbaren Netzhautrib) im ursprünglich emmetropen Auge durch Magnetextraktion eines Eisensplitters mit Sehschärfe $=1 / 3$ teilweise. Beobachtung 2 Jahre.

Fall IX. 52 jähriger Mann G. T. Aufnahme 30. III. bis 15. IV. 1905. Patient will mit beiden Augen stets gut gesehen haben. Fnde Februar 1905 angeblich Entzündung des linken Auges mit Sehverschlechterung, alle Gegenstände erschienen wie verschleiert. Vom 2. III. $1905 \mathrm{ab}$ wurde Patient von seinem Hausarzt behandelt und suchte, da keine Besserung eintrat, am 30. III. 1905 die Augenklinik auf. Er führt sein Leiden auf Erkältung zurück, zumal er seit Jahren angeblich mit chronischem Rhe u matis mus (rechtes Schultergelenk und Kreuzbeingegend) behaftet ist. - Infektion negiert.

Bei der Aufnahme am 30. III. 1905.

R. $+1,0$ D. $\mathrm{S}=5 / 5$.

L. + 1,5 D. $S=5 / 10$ teilweise (Gesichtsfeld frei).

Rechtes Auge: reizlos, normal.

Linkes Auge: starke Conjunotival- und Ciliarinjektion besonders in der nasalen Hälfte. Bulbus druckempfindlich. Im übrigen ist der vordere Bulbusabschnitt frei von Veränderungen, nux fällt an der Pupille, die rund und mittelweit ist, auf, $d a ß$ sie sich auf Atropin nicht erweitert. - Glaskörper ohne Trübungen. Ophthalmoskopisoh: Nasal und temporal unten blasige Netzhautabhebung mit stark gefüllten und geschlängelten Gefäßen. Pupille und der übrige Fundus ohne Besonderheit.

Behandlung: Natr. salicyl, heiße Umschläge.

15. IV. 1905, also nach 2 Wochen, Entlassung aus der Klinik: Die Netz* hautablösung ist restlos geschwunden.

Bei einer Nachuntersuchung nach 1 Monat lag die Netzhaut immer noch überall an. An der Stelle der ursprünglichen Ablösung zogen hinter den Retinalgefäßen und quer zu diesen einige Pigmentstreifen; zwischen denselben war das Pigmentepithel etwas entfärbt. L. $+1,5$ D. $S=5 / 10$ teilweise. 
Nachunters uchung 1914: Beiderseits blaR; vereinzelte feine Präxipitate bei sonst normalem vorderen Bulbusabschnitt.

Linkes Auge: Ophthalmoskopisch unverändert. L. $+1,5$ D. $S=5 / 75$ teilweise.

10. VII. 1918. Patient gibt an, daB er wie früher besonders bei Witterungswechsel an rheumatischen Schmerzen im rechten Schultergelenk und in der Kreuzbeingegend leide.

Beiderseits blaB; vorderer Bulbusabschnitt ohne Veränderungen (keine Präcipitate).

Linkes A uge: Die Netzhant liegt überall glatt an. In der ganzen unteren Fundushälfte and nasal oben sieht man zahlreiche kleine rundliche und einzelne verzweigte schwarze Pigmentflecke. Im Bereich der Pigmentierungen erscheint der Fundus besonders unten leicht entfärbt.

$$
\begin{aligned}
& \text { R. }+2,5 \text { D. } S=5 / 5+5,5 \text { D. } \frac{0,3}{0,4}, \\
& \text { L. }+1,5 \text { D. } S=5 / 10+4,5 \text { D. } \frac{0,23}{0,50}
\end{aligned}
$$

(Gesichtsfeld für Weis und Blau temporal um $40^{\circ}$ and oben um 30 bis $40^{\circ}$ eingeengt).

Zusammenfassung: Heilung einer partiellen blasigen Netzhautablös ung (ohne nachweisbaren Netzhautriß) im hypermetropen Auge bei nichtoperativer Behandlung mit Sehschärfe $=1 / \mathrm{g}$. An beiden Augen Iridoeyclitis rheumatica.

Beobachtung 13 Jahre.

Fall X. 27jührige Frau A. St. geb. E. Aufnahme 22. IV. bis 25. VI. 1908. Bei der Aufnahme gab Patientin an, daß sie seit 1 Jahre an Kopfschmerz über den Augen leide, zeitweise trete sogar Erbrechen auf. Vor 2 Wochen plotzlich Sehverschlechterung am linken Ange wnd vor 10 Tagen die gleichartige Sehstörung am rechten Auge, für welche Patientin keinen Grund anzugeben weiß. - Bei der Nachuntersuchung am 11. VII. 1918 wurde die Anamnese nochmals eingehend aufgenommen und hierbei stellte sich heraus, dab Patientin von Vaters Seite her aus stark rheumatisch veranlagter Familie stamme. Ihr Vater litt 14 Jahre an Gelenkrheumatismus mit Herzfehler und ist daran im Alter von 44 Jahren gestorben. 4 Brïder des Vaters sind gleichfalls Anfang der 40er Jahre an Gelentrheumatismus mit Herzfehler gestorben. Von 5 Geschwistern der Patientin starb eine Schwester im Alter von 18 Jahren an Gelenkrheumatismus mit Herzfebler, nachdem dieselbe schon als Schalkind daran gelitten haben soll. 2 Brüder sind angeblich gleichfalls mit Gelenkrheumatismus behaftet, sind zeitweise deswegen. bettlägerig, da sie alsdann nicht laufen können. - Patientin selbst hat in den letzten Jahren, besonders bei nassem Wetter, auch häufig an starken rheumatischen Schmerzen in den Kniegelenken und zeitweise bei Witterungswechsel auch an Schmerzen in den Angenhöhlen gelitten. Im übrigen fühlt sie sich jetzt (11. VII. 1918) bezüglich der Augen völlig beschwerdefrei und will wieder so gut sehen wie vor ihter Erkrankang, hat insbesondere keine hemeralopischen Beschwerden. - Patientin hat 3 gesunde Finder, 1 Kind ist mit 3 Jahren an Hirnhautentzündung gestorben. Infektion wird negiert. Die am 11. VII. 1918 ausgeführte Wassermannshe Blutserumreaktion fiel negativ ans.

Bei der Aufnahme, am 22. IV. 1908. 
St at. praes. Beiderseits geringe Ciliarinjektion, $L$ stärker als $R$; massenhaft feinste Des ce metsche Präcipitate. Pupillen mittelweit, nur sehr träge reagierend, am linken Auge unten eine Synechie. Beiderseits Glaskörper frei. - Ophthal. moskopisches Bild: Rechts und links ziemlich gleich: Papillen stark gerötet infolge stärkerer Venenfüllung, unscharfe Begrenzung. Die ganze Retina ist bis in die Peripherie hin seicht abgehoben, erscheint getrübt und undurchsichtig, so daß nirgends Aderhantzeichnung sichtbar ist; Macula lutea, relativ deutlich. Am rechten Auge sind von der Papille aus temporal 2 weiße streifige Retinalfalten mit starker Gefäßknickung bis in die Peripherie hin zu verfolgen.

Beiderseits $S=$ Fingerzählen in $10 \mathrm{~cm}$.

Interne Unters uchung: Herz und Lungen ohne Besonderheit. Steigerung allex Sehnen- und Pexiostreflexe. Rechts Andeutung von FuBklonus.

Behandlung: Beiderseits Atropin, warme Umschläge, Natr. salicyl.

Bei der Entlassung nach 2 Monaten am 25. IV. 1908 lag die Netzhaut an beiden Augen überall glatt an, desgleichen bei der Nachuntersuchung 1914, bei welcher beiderseits chorioiditische Verändexungen festgestellt wurden.

25. VI. 1908. R. $-3,0$ D. $S=5 / 15$ teilweise; $+2,0$ D. $\frac{0,20}{0,75}$.

$$
\text { L. }-3,0 \text { D. } S=5 / 10 \text { teilweise; + } 2,0 \text { D. } \frac{0,20}{0,75} \text {. }
$$

1914. R. E. $S=6 / 6 \frac{0,30}{0,30}$,

$$
\text { L. E. } S=6 \frac{0,30}{0,30} \text {. }
$$

Bei dex letzten Nachuntersuchung am 11. VII. 1918. Beider seits blaß; keine Präcipitate, keine Synechien, Glaskörper frei.

Rechtes Auge. Ausgesprochene Chagrinierung des ganzen Fundus; kleine hellrötliche und gelbliche chorioiditische Herdchen sowie rundliche Pigmentflecke, die schon an der Papille begimen und überall bis in die äußerste Peripherie verstrent sind. Die Papille selbst ist circulär von unregelmäBiger alter chorioiditischer Pigmentierung umgeben; nasal von der Papille liegt ein größerer Entfärbungsbezirk, der ohne scharfe Grenze in die Umgebung übergeht. Beim Blick peripher unten bemerkt man eine große Zahl größerer alter meistenteils schwarz pigmentierter' chorioiditiseher Herde.

Linkes Auge: gleichfalls abgelaufene circumpapilläre Chorioiditis in Form eines breiten Entfärbungsringes mit unregelmäBiger Pígmentierung; anschließend eine ziemlich breite Entfärbungszone, die ohne scharfe Grenze in die Umgebung übergeht. Fundus wie rechts ausgesprochen chagriniert und nit zahllosen meist etwas größeren pigmentierten chorioiditischen Herden versehen. Stellenweise sind die Netzhautgefäße mit Pigment eingescheidet, besonders ist eine nasal gelegene Vene von der Papille an auf größere Strecke pigmentiert. Wie rechts sind die chorioiditischen Herde peripher unten am zahlreichsten und größten. An einer Stelle nasal unten ausgesprochene netzförmige Retinalpigmentierung.

Beiderseits E. $\$=5 / 4$ teilweise $\frac{0,30}{0,40}$.

Am rechten Auge Gesichtsfeld für Weiß bei Tageslicht und bei stark herabgesetzter Beleuchtung gleichmäßig oben um $20^{\circ}$, temporal um 10 bis $15^{\circ}$, für Blau oben, unten und am stärksten temporal eingeengt. 
Am linken Auge Gesichtsfeld für WeiB bei Tageslicht und bei stark herab. gesetzter Beleuchtung annähernd normal, für Blau stark konzentrisch eingeengt.

Zus ammenfass ung: Heilung einer doppel seitigen totalen seichten Netzhautablös ung (ohne nachweisbaren Netzhautriß) i m e mmetropen Auge bei nichtoperativer Behandlung mit Sehschäre $=5 / 4$ teilweise. Atiologie zweifellos rhe matis ch exs udative Chorioiditis (Iritis aerosa). Ausgang in Chor ioretin is disseminata.

Beobachtung 10 Jahre.

Fall XI. 36jähriger Mann G. R. Aufnahme 3. V. bis 14. V. 1910.

Patient wurde 1905 wegen akuter Tenonitis (mit starker Chemosis) des rechten und wegen rückgängiger Tenonitis des linken Auges mit Natrium salicyl. 2 Wochen lang ambulatorisch behandelt. Die Augen blieben gut bis Februar 1910; danals trat gleichzeitig mit Gliederschmerzen, Schüttelfrost und Hitze eine ähnliche schmerzhafte Schwellung beider Augen auf wie 1905; die Augen konnten angeblich nicht geöffnet werden. Ostern 1910 wieder Gliederschmerzen mit Schwellung und Schmerzhaftigkeit des linken Auges von 4tägiger Dauer. Seitdem soll das linke Auge häufiger rot sein bei gleichzeitigem Kopfschmerz. Seit 2 Wochen Sebverschlechterung am linken Auge. - Seit 1898 hat Patient fünfmal schweren Gelenkrheumatismas durchgemacht, mußte jedesmal 17-18 Wochen mit hohem Fieber zu. Bett liegen, zuletzt 1909; damals wegen gleichzeitiger Pleuritis und Perikarditis 24 Wochen bettlägerig; Punktionen waren nioht orforderlich - trotz großer Ergüsse. Im übrigen ist Patient angeblich gesund, die Familienanamnese belanglos.

Stat. pr. Rechtes Auge normal.

R. + 2,0 D. cyl. A. $0^{\circ} \mathrm{S}=5 / 5$ teilweise; $\frac{0,28}{0,40}$.

Linkes Auge: Mäßige Giliarinjektion; umschriebene episkleritische In. jektion in der Aquatorgegend temporal und oben. Pupille ohne Besonderheit.

ophthaImoskop isch: Flache, durchsichtige Netzhautablösung, die am nasalen Papillenrande beginnt und nasal unten in eine scharf umschriebene blasige Abhebung von grauschwarzer Farbe übergeht, an deren Rande man einen langen senkrecht zu den NetzhautgefäBen verlaufenden Pigmentstreif bemerkt. Ganz peripher unten wird die Netzhautablösung wieder flacher und etwas durchsichtiger.

L. $S=1 / 25$. Gesichtsfeld oben um $30^{\circ}$ und temporal um $10^{\circ}$ eingeschränkt.

Behandlung: 11 tägige Schwitzkur; Aspirin 2-3 g.

Bei der Entlassung am 14. V. 1910 war die Netzhautablösung etwas zurückgegangen.

Januar 1914 gab Patient bei der Nachuntersuchung an, daß er in den letzten 4. Jahren noch 2 mal, also seit $1898 \mathrm{im}$ ganzen 7 mal an schwerem Gelenkrheumatis mus erkrankt war.

1914 lag die Netzhaut im linken Ange überall an, das Gesichtsfeld war normal, Sehschärfe beiderseits $=5 / 4$.

Am 4. VII. 1918.

R. $+1,5$ D. cyl. A. $0^{\circ} \mathrm{S}=5 / 4 ;+1$ D. $=$ cyl. $+1,5 \ldots \frac{0,30}{0,40}$,
L. +1 D. cyl. A. $0^{\circ} 1=5 / 4 ;+1$ D. 0 cyl. +1 D. . $\frac{0,30}{0,40}$.

Gesichtsfeld normal. 
Reehtes Auge: normal.

Li nkes Auge: Vorderer Bulbusabschnitt normal. Ophthalmos ko p isch: Die Netzhaut liegt überall glatt an; im nasalen Abschnitt sowie unten und peripher temporal oben feinfleckige rundliche Pigmentierungen, die nasal unten am stärksten sind; nasal oben ein langer feiner Pigmentstreif.

Zusammenfassung: Heilung einer ausgedehnten, stellenweise blasigen Netzhautablös ung (ohne nachweisbaren Netzhautriß) in einem Auge mit geringem h y peropischen As tigmatis mus bei nichto perativer Behandlung mit Sehschärfe $=5 / 4$. Ätiologie zweifellos rheumatische exsudative Chorio iditis (Tenonitis, Episkleritis). A usgang in Chorioiditis disseminata. Beobachtung 8 Jahre.

Fall XII. 37 jähriges Fräulein El. Sch. Aufnahme 17./VIII. bis 17. IX. 1903. Patientin bemerkte am 26. VII. 1903 plötzlich eine Verdunkelung am rechten Auge „wie eine Wolke ${ }^{\iota}$, für welche sie keine Ursache anzugeben weiß. Beide Augen sind angeblich stark kurzsichtig, und zwar soll sich die Kurzsichtigkeit allmählich nach einer doppelseitigen Regenbogenhautentzündung entwickelt haben, an weleher Patientin rom 9. III. bis 10. IV. $1886 \mathrm{klinisch}$ behandelt wurde. Im damaligen Krankenblatt ist notiert: Beiderseits Iritis serosa, Präcipitate, flottierende Opacitates corp. vitr. - Behandlung mit Pilocarpininjektionen und Sublimat-Aloepillen. Im Entlassungsstatus vom 10. IV. 1886 ist notiert:

R. $S=6 / 60$. L. $S=8 / 24$ ? Von einer Myopie ist nichts erwähnt.

Bei einer nochmaligen genauen Aufnahme der Anamnese im Juli 1918 gab Patientin an, daß sie schon seit vielen Jahren an starkem Rheu matis mus in den Beinen leide und daß sie in letzter Zeit sowie gegenwärtig von rhe u matischen Schmerzen in den Händen geplagt sei. - Im übrigen ist Patientin angeblich gesund; für Lues, Tuberkulose usw. kein Anhaltspunkt. Wasser mann. sche Reaktion (10. VII. 1918) negativ.

Stat. praes. (17. VIII. 1903). Rechtes Auge reizlos; o phthalmosko. pis ch: vereinzelte Glaskörpertrübungen. Der Fundus erscheint mäßig verschleiert. Papille unscharf begrenzt, mit temporaler Sichel von mäBiger Breite. Papillenbreit unterhalb der Papille ist die Retina in Form einer steilen Blase abgehoben, welche fast die ganze untere Fundushälfte einnimmt. Die Maculagegend ist nur flach abgelöst und die Netzhaut erscheint hier grauweiB getrübt. In der ganzen oberen Hälfte sieht man die Chorioidealzeichnung deutlich. R. $-7,0$ D. $S=5 / 35$, o. Gl. $\frac{0,11}{0,75}$ mühsam. Das Gesichtsfeld ist oben um $40^{\circ}$ eingeschränkt.

Linkes Auge: Außerlich normal; ophthalmoskopisch: mäBige temporale Sichel; 3 kjeine gelbliche alte chorioiditische Herde in der Macula lutea

$$
\text { L. }-12,0 \text { D. } \mathrm{S}=5 / 15 \text { fast; o. G1. } \frac{0,09}{0,30} \text {. }
$$

Behandlung: 18. VIII. 1903. Netzhautdurchschneidung am rechten Auge nach Deutschmann (Prof. E. v. Hi p pel). Rasche Rückbildung der Netzhautablösung; 2 Tage nach der Operation war die Netzhaut wieder angelegt. Bei der Entlassung nach ei ne m Monat war nur noch ganz pexipher innen unten eine Andeutung von Netzhauttrübung vorhanden. Die Sehschärfe hatte sich nicht gebessert, das Gesichtsfeld war etwas weniger eingeengt.

Bei der Nachuntersuchung 1914 lag die Netzhaut des rechten Auges überall an.

$$
\text { R. }-14,0 \text { D. } S=5 / 10 ;-8,0 \text { D. } \frac{0,15}{0,40} \text {. Gesichtsfeld normal. }
$$




$$
\text { L. }-10,0 \text { D. } S=5 / 5,5 ;-7,0 \text { D. } \frac{0,25}{0,40} \text {. }
$$

Bei der Nachuntersuchung am 10. VII. 1918. Patientin sieht seit 6-8 Wochen mit dem rechten Auge angeblich Regenbogenfarben um Lichter.

Stat. praes.: Reehtes Auge. Deutliche Kerntrübung und pexiphere Linsentrübungen. Einzelne feine fädige Glaskörpertrübungen. Mäßig breites ringförmiges Staphyloma posticum. Feiner welliger gelblicher Aderhatatstreif in der Manula lutea. Peripher unten, besonders temporal mehrere größere gelblich weiBe alte Aderhautherde, wahrscheinlich dem Operationseinstich entsprechend; nasal unten sieht man einen weißlichen Streif, der wahrseheinlich dem Ausstich entspricht. Die Retina liegt äberall glatt an. $T_{2}=18 \mathrm{~mm}$ Hg.

$$
\text { R. }-14,0 \text { D. } S=5 / 25 \text { nahezu; }-8,0 \text { D. } \frac{0,10}{0,40} \text {. Gesichtsfeld normal, abge- }
$$

sehen von geringer Finschränkung oben bei herabgesetzter Beleuchtung.

Linkes Auge unverändert, L. - 10,0 D. S $=5 / 7,5$ teilweise; $-7,0$ D. $\frac{0,27}{0,40}$.

Einzelne Fingergelenke beider Hände a ufällig verdickt.

Zusammenfassung: Heilung einer ausgedehnten blasigen Netzhautablös ung (ohne nachweisbaren NetzhautriB) in einem hochgradig myopischem Auge bei operativer Behandlung mit Sehschärfe $=1 / 2$, die jedoch infolge beginnender Katarakt anf etwa $1 / 5$ gesunken ist, Rhe u matis ehes Individuum, das 17 Jahre vor der Netzhautablösung eine doppelseitige Iritis serosa überstanden hat. Beobaoktung is Jahre.

DaB in all diesen Fällen mit Fug und Recht von „Heilung" gesprochen werden darf, geht aus der Dauer der Beobachtung ohne weiteres hervox. Die jüngste Beobachtung erstreckt sich auf $2 \mathrm{Jahre}$, die nächste a uf 4 Jahre, drei weitere auf 6,8 und 10 Jahre, einmal wurde die Heilung 13 Jahre, zweimal 15 Jahre und in einem Falle 16 Jahre hindurch verfolgt. - Hervorheben möchte ich, daß es sich nicht nur um anatomische, sondern bei allen Kranken um f unktionelle Dauerheilungen handelt, da sämtliche ein brauchbares, einzelne sogar ein ausgezeichnetes Sehvermögen zurückbehielten. Nur bei einer Patientin ist anzunehmen, daß die Sehschärfe vielleicht weniger als $1 / 12$ der Norm betrug; es ist das Fall $V$ (dop pelseitige Ablatio ret., doppelseitige Heilung), in welchem eine Nachuntersuchung leider nicht möglich war, in welchem aber durch genaues Befragen des gleichfalls an Netzhautablösung erkrankten und geheilten Sohnes festgestellt werden konnte, daß die Mutter sich nicht nur überall sicher orientiere, sondern sogar die im Haushalt exforderlichen Näharbeiten verrichte. - Der Sohn behielt ein Sehvermögen von $1 / 12^{\circ}$ Einmal betrug die Sehschärfe $=1 / 10$, ein mal $=1 / 4$, dreimal $=1 / 3$, z weimal $=1 / 2$ und dreimal $=11 / 4$ (in Fall $X$ sogar beiderseits $S=11 / 4$ ).

Unter diesen 12 geheilten Fällen befinden sich 4 Kranke mit doppelseitiger Netzhautablösung; bei zweien von diesen (Fall VI und $\mathrm{X}$ ) trat an beiden Augen Heilung ein (also Heilung an im ganzen 14 Augen), 
während bei den anderen beiden (Fall IV und VII) nur ein Auge heilte und der Ausgang am zweiten Erblindung (Cataracta complicata) war.

Da die in Rede stehende Statistik, wie eingangs erwähnt wurde, 163 Patienten bzw. 186 Augen umfaßt, so bedeutet dies: Heilung der Netzhautablösung in 7,5\% der Fälle (und Augen).

Genau in der Hälfte der geheiten Fälle lag stärkere bzw. hochgradige Myopie vor; nur einmal eine Myopie von 7, o D., während bei den 5 anderen die Myopie 14,0 bis 19,0 D. betrug. - Aber nur bei 4 (Fälle I-IV) von diesen 6 Myopen kann meines Erachtens die Myopie mit genügender Sicherheit auch als Ursache für die Entstehung der Netzhautablösung angesprochen werden. In Fall $\mathrm{V}$. handelt es sich zwar um einen Myopen von beiderseits - 15,0 D., für die Beurteilung der Ätiologie ist es jedoch zweifellos recht wichtig, daß der Kranke 4 Jahre vor Eintritt der Ablösung an doppelseitiger Keratitis parenchymatosa und Chorioiditis disseminata, also an einer exsudativen Entzündung behandelt wurde. (Wassermannsche Reaktion war negativ!) Mindestens ebenso fraglich erscheint mir der ursächliche Zusammenhang zwischen Myopie und Ablatio in dem in der Literatur ${ }^{1}$ ) häufiger zitierten Fall XII (R-14,0 D.). Hier war der Netzhautablösung 17 Jahre zuvor eine doppelseitige Iritis serosa voraufgegangenen - nach der Anamnese und wegen der Verdickungen der Fingergelenke bei negativer Wassermannseher Reaktion sicherlich rhe umatischer Natur - also auch hier eine exsudative Entzündung im Bereich der Uvea. Auffallend ist noch in diesem Falle die Angabe der Patientin, daß sich ihre Kurzsichtigkeit erst nach der Iritis, d. h. nach dem 20. Lebensjahr entwickelt habe. Einer solchen Angabe würde man keine allzu große Bedeutung beimessen dürfen, wenn sie nicht mit dem Befunde der Krankengeschichte in Einklang stände, die anläßlich der klinischen Behandlung der Iritis serosa (1886) angefertigt wurde: Visus $R=6 / 60$; $\mathrm{L}=6 / 24$ ?, von Myopie nichts erwähnt. - Gegen die Annahme der Myopie als alleiniges ätiologisches Moment für die Ablatio scheint mir ferner zu sprechen, daß im vorliegenden Falle im Glaskörper nur leichte Veränderungen gefunden wurden und da $B$ die für hochgradige Kurzsichtigkeit $(\mathrm{R}-14,0$ D.) charakteristischen Veränderungen an der Papille und in der Macula lutea gleichfalls nur gering waren (p. 87). Wo Myopie die Ursache' für die Netzhautablösung abgibt, sind aber in der Regel schwere Veränderungen, wenn auch nicht immer im Glaskörper nachweisbar, so doch an der Netzhaut-Aderhaut vorhanden. - Selbstrerständlich soll nicht in Abrede gestellt werden, daß in diesen beiden Fällen der myopische

1) Bericht über die XXXI. Vers. d. ophth. Ges. 1903, S. 23. E. v. Hi p pels Diskussionsbemerkung (Fall II) zu Uhthoffs Vortrag ,Zur Wiederanlegung der Netzhautablösung “‘. 
Bau des Auges den Eintritt der Netzhautablösung begünstigt habe. Als ausschlaggebendes Moment muß aber meines Erachtens eine exsudative Chorioiditis gelten, deren Ätiologie in Fall $V$ unbekannt, in Fall XII ein chronischer Rheumatismus ist.

Sicherlich ist es nicht nur von theoretischem, sondern unter Umständen von großem praktischen Interesse, wenn wir uns in jedem Falle von sogenannter Myopie-Ablatio darüber Rechenschaft ablegen, ob die Ablösung durch ",Zug von innen her" oder durch einen „primären subretinalen Erguß “ bewirkt wurde. Denn unsere Statistik bestätigt es von neuem, daß die Prognose der durch Exsudation bedingten Ablatio relativ viel besser ist als diejenige der durch Zugwirkung entstandenen. Unter den 163 Fällen bzw. 186 Augen mit Netzhautablösung sind 62 (Fälle) bzw. 69 (Augen) mit Myopie als Ursache der Erkrankung; von diesen sind 4 geheilt, d.h. Heilung der MyopieAblatio in $6,4 \%$ der Fälle bzw. in 5,8\% der erkrankten Augen.

Wie stellt sich nun demgegenüber die exsudative Form der Ablatio? - Unsere Statistik enthält 17 derartige Fälle bzw. 19 solche Augen; unter diesen sind 5 Heilungen, davon eine doppelseitig $=6$ geheilte Augen, d.h, Heilung der durch primären,subretinalen ErguB bedingten Netzhautablösung in $29,4 \%$ der Fälle bzw. in $31,6 \%$ der erkrankten Augen.

Recht bemerkenswert erseheint es mir, daß unter den 5 geheilten Faillen von exsudativer Form der Ablatio nicht weniger als 4 mal , $\mathrm{Rh}$ h u -

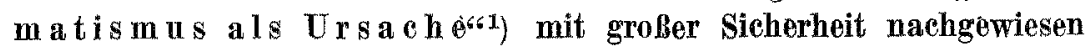
werden konnte (in Fall $V$ blieb die Ursache unbekannt), und zwar kann sowohl der chronische Rheumatismus wie der akute Gelenkrheumatismns zur Netzhautablösung führen - in gleicher Weise, wié wir das von der rheumatisehen Iritis und anderen rheumatischen Augenafiektionen her wissen. - Für die rheumatische Basis der Netzhautablösung ist insbesondere die Anamnese des Falles $X$ sehr charakteristisch: die mit chronischem Rheumatismus behaftete Frau (beiderseits Ablatio retin. und Iritis serosa) verlor ihren Vater in jungen Jahren an

1) Aus praktischen Grinden darf an dieser Stelle wohl von , Rheumatismus als Ursache" gesprochen werden. Doch bleibe man sich dessen bewußt, daß die Bezeichnung Rheumatismus, wie die jüngsten Arbeiten von neuem betonen, nur ein Sammelbegriff ist, unter dem eine Anzahl von Erkrankungen zusammsngefaßt werden, welche bei oft geringfügigen anatomischen Veränderungen mit großen. Schmerzen und dadurch mit Funktionsstörungen im Bewegungsapparat einhergehen. Ursächlich pflegt die Errkranknng mit Erkältung und atmosphärischen Einflüssen in Beziehung gebracht zu werden. Tat. sächlich ist also Rhetuatismus weder eine ätiologisohe noch klinisehe Hinheit. H. Quineke, "Über Rheumatsmus". Deutsehe med. Wochenschr. 1917, Nr. 32 und 39 . E. Roos, ,Über Rheumatismus ${ }^{a s}$. Münch. med. Wochenschr. 1920, Nr. 4 . 
Gelenkrheumatismus mit Herzfehler (derselbe hatte 14 Jahre lang wiederholt schwere Attacken). An dem gleichen Leiden starben 4 Brüder des Vaters und eine 18 jährige Schwester der Patientin; ihre beiden Brüder sind gleichfalls Rheumatiker und häufiger infolge von chronischem Gelenkrheumatismus bettlägerig. - Die Bedeutung des akuten Gelenkrheumatismus als Ursache der Netzhautablösung ist in äuBerst typischer Weise durch Fall XI illustriert: innerhalb von 20 Jahren hatte der Kranke 7 schwere Attacken von akutem Gelenkrheumatismus und zwar meist von monatelanger Dauer; eine solche war durch Pleuritis und Pericarditis mit großen Ergüssen kompliziert. Wiederholt waren die Augen in Form von Tenonitis mitbeteiligt und in einem Anfall entwickelte sich bei gleichzeitiger leichter und flüchtiger Episkleritis eine Netzhautablösung.

Es ist auffallend, daß der rhe $\mathrm{matischen}$ Grundlage der Netzhautablösung bisher meines Wissens nirgends Erwähnung getan wurde. In den aus der Uht hoffschen Klinik hervorgegangenen sorgfältigen und umfassenden klinisch-statistischen Mitteilungen über Netzhautablösung von Müglich') und $S p a m e r^{2}$ ) ist der Rheumatismus als Ursache nicht genannt und selbst in Lebers klassischer Bearbeitung der Netzhautablösung (Gräfe-Saemisch, Handb. der Augenheilk., II. Aufl., 1916) ist nur an einer Stelle (S. 1498) gelegentlich der Besprechung des Falles IX dieser Arbeit kurz angegeben: ,Klagt über viel "Rheumatismus" in den Extremitäten und im Kopf." Auch in dem typischen Falle $X$, der - allerdings unter anderem Gesichtspunkte auch schon von Leber zitiert wurde, blieb ihm die rheumatische Ätiologie unbekannt, da im klinischen Krankenblatt ursprünglich die Familienvorgeschichte nicht berücksichtigt war und Pat. auch von ihrer eigenen rheumatischen Erkrankung, die sie für das Augenleiden als bedeutungslos betrachtete, erst Mitteilung machte, als sie von mir danach gefragt wurde.

Daß ich in der Lage bin, aus dem nicht allzu großen Material vier Fälle von geheilter rheumatischer Netzhautablösung mitzuteilen, ist doch schwerlich nur ein glücklicher Zufall. Es wäre jedenfalls wünschenswert, wenn künftig die geheilten Fälle, aber - in Rücksicht auf Prognose und Behandlung - auch alle anderen Netzhautablösungen vom Gesichtspunkte eventueller rheumatischer Entstehung eingehend untersucht würden. Für diese Form der Netzhautablösung ist es anscheinend charakteristisch, da $B$ sie nicht als isolierte entzündliche Erscheinung der Aderhaut auftritt, sondern

1) Müglich, Übex Spontanheilung der Netzhautablösung. Inaug.-Dissert. Marburg 1891.

2) S pamer, Über Netzhautablösung mit besonderer Berücksichtigung der Wiederanlegung derselben. Inaug.-Dissert. Breslau 1904. 
da $B$ derselben andere Entzünd ungen am $A$ uge (Iritis, Cyclitis, Episkleritis, Tenonitis) voraufgehen oder sie begleiten.

Bezüglich ihrer Ätiologie blieben noch 3 unserer 12 Fälle zu besprechen, von denen der eine (Fall VIII) traumatischer Art ist und bezuglich seiner Entstehung und seines Verlaufs keine Besonderheit bietet. Um so interessanter sind dagegen die Fälle VI und VII, do p pelseitige Netzhautablösung bei Mutter and Sohn in nichtmyopischen Augen. Bei hochgradiger Myopie wäre ein derartig familiäres Auftreten der Erkrankung ohne weiteres verständlich; in den vorliegenden Fällen geben uns leider weder die Vorgeschichte noch der objektive Befund irgendeinen ätiologischen Anhaltspunkt. Das Merkwürdige der Beobachtung, die ein Unikum darstellt, liegt ja nicht nur in dem familiären Auftreten der Netzhautablösung bei Nichtmyopie, sondern weit mehr in der Heilung derselben bei Mutter und Sohn, die bei der Mutter sogar an beiden Augen erfolgte.

Es muß hervorgehoben werden, daß die Erkrankung in der Mehrzahl der Fälle von vornherein prognostisch durchaus ungünstig erschien. Handelte es sich doch nur vereinzelt um kleine umschriebene Abhebungen, meist waren dieselben sehr ausgedehnt und blasig oder faltig; nur in einem Falle allerdings war eine totale (aber seichte) Ablösung an beiden Augen vorhanden.

Bemerkenswert ist, daß unter den geheilten Augen - trotz wiederholter, speziell darauf gerichteter Untersuchungen bei weiter Pupille - nur ein malein Netzha utriß nachgewiesen werden konnte; es ist das Fall $\mathrm{I}(\mathrm{R}-19,0$ D.), bei dem die Ablösung sich auf die nächste Umgebung des Risses beschränkte. In Fall IV von doppelseitiger Ablatio (R. Myopie unbekannten Grades; $\mathrm{L}-7,0$ D.) , waren am rechten Auge, das der Frblindung anheimfiel, 2 Netzhautrisse vorhanden, wogegen am geheilten linken kein Riß nachgewiesen werden konnte. Ich möchte dies erwähnen, obne dem Vorhandensein eines Risses einen prognostischen Wert beimessen zu wollen, zumal wir ja gar nicht wissen toönen, wie oft ganz periphere Netzhautrisse unserer ophthalmoskopischen Untersuchung unzugänglich sind. Ergänzend sei hinzugefügt, daß unter sämtlichen 163 Fällen unserea Statistik 19 mal Netzhautrisse (= 11,6\%) festgestellt wurde, davon $9 \mathrm{mal}$ bei Myopie und $10 \mathrm{mal}$ bei Nichtmyopie. Die Zahl der myopischen Augen mit Netzhautablösung betrag 105, diejenige der nichtmyopischen 81. Auffallenderweise komnten also bei Nichtmyopie häufiger $(=12,3 \%)$ Netzhautrisse nachgewiesen werden als bei Myopie $(=8,6 \%$ ), eine Tatsache, in der ich nur den Beweis erblicken kann, daß die Lebersche Lehre von der (häufigsten) Entstehung der Netzhautablösung, ,durch Zug von innen her" für einen großen Teil der nichtmyopischen Ablatio ebenso zutrifft wie für die myopische. 
Aus den mitgeteilten Krankengeschichten geht hervor, daß die Art der Behandlung im allgemeinen eine konservative und die Dauer des klinisehen Aufenthalts zum Teil sehr kurz und niemals übermäßig lang (11-46 Tage) war. In üblicher Weise wurde für die ersten Tage meist Bettruhe angeordnet; daneben wurden Quecksilber- und Schwitzkuren, Sublimatpillen, Jod- und Salicylpräparate und Iokal vor allem subconjunctivale Kochsalzeinspritzungen, hie. und da auch nur heiße Umschläge angewandt. Über die Wirkung der einzelnen Maßnahmen ließ sich kein sicheres Urteil gewinnen. Nur sei hervorgehoben, daß die rheumatischen Netzhautablösungen prompt auf Schwitzkur, Salicylpräparate und heiße Umschläge sich besserten sehr begreiflich, da hier die die Netzhautablösung bewirkende subretinale Flüssigkeit keine andere Stellung einnimmt, wie die rheumatischen Ergüssein den Gelenken, in Perikard und Pleura. Die Analogien zwischen Uvea einerseits und Synovialmembran und den serösen Häuten der Körperhöhlen anderseits sind ja auch sonst aus der Pathologie genügend bekannt.

Nur 3mal war die Behandlung eine operative. Selbstverständlich wurde bei der traumatischen Ablösung, die durch einen intraocularen Eisensplitter bedingt war, die Magnetextraktion ausgeführt. - In Fall VI von familiärer Ablatio wurden neben anderem zwei Skleralpunktionen gemacht. Die Heilung trat hier, wie übrigens in dem zugehörigen rein konservativ behandelten Fall von familiärer Ablatio, erst so lange nach der Entlassung aus der Klinik auf, daß von einem therapeutischen Effekt kaum gesprochen werden darf, die Fälle vielmehr als Spontanheilungen betrachtet werden müssen. - SchlieBlich wurde in Fall XII die Netzhautdurchschneidung nach Deutschmann ausgeführt. Die auffallend rasche Heilung, die schon 2 Tage nach dem Eingriff eintrat, schien bisher die ZweckmäBigkeit dieses Verfahrens besonders darzutun, zumal es sich um eine blasige Netzhautablösung bei einer Myopie von 14,0 D. handelte. Nachdem aber, wio ich hoffe, mit genügender Beweiskraft gezeigt werden konnte, daß in diesem Falle die Ablösung nicht aus myopischer, sondern aus rheumatischer Ätiologie, also nicht durch "Zug von innen her", sondern durch einen

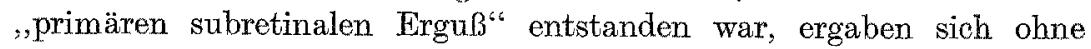
weiteres Bedenken, ob bier wirklich die Operation ein ausschlaggebender Heilfaktor war und ob nicht vielmehr die Heilung auch bei der üblichen konservativen antirheumatischen Behandlung in ähnlicher Weise exfolgt wäre, wie wir es in analogen Fällen gesehen haben? - Kam in diesem Falle (die Netzhautablösung war nur etwa 3 Wochen alt und in keiner Weise vorbehandelt) der Operation wirklich eine Heilwirkung zu, was nicht in Abrede gestellt werden soll, so war es doch lediglich der Effekt der Exsudatablassung, der auch durch die gefahrlosere Sklerochorioidealpunktion zu erzielen gewesen wäre. 
So sehr die operativen Bestrebungen bei der im allgemeinen doch recht ungünstigen Prognose zu begrüßen sind, so werden wir - das zeigen die vorliegenden Beobachtungen - bei der rhe umatischen Netzhautablösung zurückhaltender sein dürfen. Eine Operation (zunächst Sklerochorioideal punktion) ist hier erst dann gerechtfertigt, wenn eine genügend lang fortgesetzte nichtoperative Lokal- und antirheumatische Allgemeinbehandlung versagen sollte.

\section{Druckfehler-Berichtigung.}

Auf Seite 314 meiner letzten Arbeit in diesem Archive, Bd.102, Heft $3 / 4$ (Die Bedeutung der Diffraktion für das Problem etc.) sind versehentlich in $A b s a t z 3,4$ und 5 statt der richtigen Ausdrücke "Absorption" und "absorbiert" die Druckfehler "Adsorption" und ,adsorbiert" gesetzt worden.

L. Koeppe, Halle a. S. 